\title{
Incapacidade para o trabalho: análise dos benefícios auxílio-doença concedidos no estado de Rondônia
}

\author{
Inability to work: analysis of sickness benefits granted \\ in the State of Rondônia
}

Heinz Roland Jakobi ${ }^{1}$
Anadergh Barbosa-Branco ${ }^{1}$
Luis Fernando Bueno ${ }^{2}$
Ricardo de Godoi Mattos Ferreira ${ }^{3}$
Luís Marcelo Aranha Camargo ${ }^{4}$

${ }^{1}$ Programa de Pós-

Graduação em Ciências da

Saúde, Departamento Saúde

Coletiva, Universidade de

Brasília. Campus

Universitário Darcy Ribeiro

Asa Norte Faculdade de

Ciências da Saúde. 70.910-

900 Brasília DF Brasil.

heinzjakobi@hotmail.com

${ }^{2}$ Universidade Federal de

Rondônia.

${ }^{3}$ Fundação Osvaldo Cruz.

${ }^{4}$ Universidade de São Paulo.

\begin{abstract}
This is a descriptive epidemiological study aiming to o estimate the prevalence and $d u-$ ration of sickness benefits granted by the Brazilian Social Security Institute, as well as the clinical causes of inability to work among employees in Rondonia in 2008. The prevalence was submitted by 10,000 workers and stratified by sex, age, diagnosis, economic activity, and type and duration of benefits. Men had a higher prevalence and duration of benefits. Social security benefits were found to be 2,5 times more prevalent than accident benefits and both increased with age. Labor accidents were more prevalent in the age group ranging between 50-59 years, and forestry, construction and manpower leasing had the highest prevalence. The main diagnostic groups were injuries, musculoskeletal system disorders and digestive diseases. The duration of the sickness benefits was higher among men, $=40$ years and in the social security category. The economic activity is an important risk factor for inability to work, and it needs to be further explored in order to improve sickness prevention and reduce the impact on workers' health arising from environmental conditions and working processes.
\end{abstract}

Key Words Social security, Forestry, Injuries, Rondônia
Resumo Trata-se de um estudo epidemiológico descritivo com objetivo de estimar a prevalência e a duração dos benefícios auxílio-doença, concedidos pelo Instituto Nacional de Seguro Social, bem como dos fatores associados à incapacidade para o trabalho entre os trabalhadores empregados em Rondônia em 2008. As prevalências foram apresentadas por 10.000 trabalhadores e estratificadas por sexo, idade, diagnóstico, atividade econômica, espécie e duração dos benefícios. Os homens apresentaram maiores prevalência e duração dos benefícios. Os benefícios previdenciários mostraramse 2,5 mais prevalentes do que os acidentários e ambos aumentaram com a idade. Os acidentários foram mais prevalentes na faixa etária de 50-59 anos. Silvicultura, construção civil e locação de mão de obra apresentaram as maiores prevalências. Os principais grupos diagnósticos foram lesões; doenças do sistema osteomuscular e doenças do aparelho digestivo. A duração dos benefícios auxílio-doença foi maior entre homens, com $\geq 40$ anos e na espécie previdenciária. A atividade econômica é um importante fator de risco para a incapacidade para o trabalho, e precisa ser mais bem explorada como tal visando melhorar a prevenção de doenças e diminuir o impacto na saúde dos trabalhadores decorrentes das condições ambientais e dos processos de trabalho.

Palavras-chave Previdência social, Silvicultura, Lesões, Rondônia 


\section{Introdução}

A incapacidade para o trabalho possui implicações sociais, econômicas e para a saúde pública. Neste contexto, diversos são os aspectos a serem considerados. Entre eles destaca-se o fato de que $o$ afastamento por doença tem um efeito negativo sobre as empresas e a economia em geral, resultando na redução da força de trabalho e no aumento do custo da produção, médico, previdenciário e social ${ }^{1}$. Os danos à saúde do trabalhador vêm acompanhando o processo de crescimento socioeconômico e industrial mundial, assim como a consequente diversificação dos processos produtivos, os quais expõem os trabalhadores a riscos ambientais. Este fato dá margem a agravos à saúde que podem levar à incapacidade para o trabalho ${ }^{2}$. No estado de Rondônia, a atual fase de ocupação, chamada de desenvolvimento agroindustrial, florestal ou agronegócio, aponta para a presença de situações específicas de riscos vinculados ao processo produtivo regional, ou seja, ao desenvolvimento agropecuário ${ }^{3-8}$. Estudo anterior sobre os riscos ocupacionais mais prevalentes no estado de Rondônia identificou forte tendência na atividade de produção agropecuária, totalizando $73,0 \%$ das empresas; seguido do comércio, com 19,2\%; da indústria, em terceiro, com $4,3 \%$; e do setor de serviços, que conta com apenas 3,5\% de participação econômica. No setor agropecuário, há grande participação da criação de bovinos de corte e produção de leite $(54,0 \%)$, enquanto na agricultura $10,8 \%$ das empresas estão voltadas para o cultivo de café. Entre as empresas rondonienses prevalecem as de grau de risco 3 (78,6\%), o que por si só já caracteriza o elevado risco ocupacional das atividades desenvolvidas por essas empresas e seus empregados ${ }^{8}$. No setor agrosilvopastoril, observa-se a exposição aos riscos e às enfermidades no campo, no trabalho rural. Observa-se agravos à saúde do trabalhador decorrentes de infecção de vias aéreas superiores, acidentes ocasionadas pelos desmatamentos e queimadas; insolação, queimaduras e câncer de pele devido à radiação solar; doenças ergonômicas: lesões por esforços repetitivos (LER) $e$ doenças osteomusculares relacionadas ao trabalho (DORT); intoxicação e câncer devido à exposição a agrotóxicos; acidentes mecânicos; acidentes pérfuro-cortantes; doenças infecciosas e parasitárias como malária, tuberculose, leishmaniose, doença de Chagas $^{8}$.

As estatísticas oficiais no Brasil sobre a incapacidade para o trabalho são limitadas aos trabalhadores segurados - empregados, autônomos, e demais contribuintes da Previdência Social. Não há dados oficiais para o grande número de trabalhadores informais. O Ministério da Previdência Social possui o maior banco de dados referente a características demográficas e de saúde de uma parcela significativa da população trabalhadora brasileira, no entanto, esses dados são pouco explorados do ponto de vista epidemiológico.

A análise das características dos benefícios auxílio-doença fazem deles um bom indicador das condições de saúde/doença da população adulta trabalhadora ${ }^{1}$.

Este estudo objetivou analisar os agravos à saúde que levam à incapacidade para o trabalho na população empregada em Rondônia, gerando a concessão de benefícios auxílio-doença por parte do Instituto Nacional de Seguridade Social (INSS) da Previdência Social (PS).

\section{Método}

Trata-se de um estudo epidemiológico descritivo contemplando todos os trabalhadores empregados que obtiveram benefícios auxílio-doença pelo INSS em Rondônia no ano de 2008.

Os benefícios auxílio-doença foram analisados considerando as seguintes variáveis: causa clínica da incapacidade (Classificação Internacional de Doenças - CID-10, grupo, subgrupo e causa específica) ${ }^{9}$, espécie de benefício (auxíliodoença previdenciário, auxílio-doença acidentário), ramo de atividade econômica (Cadastro Nacional de Atividades Econômicas - CNAE divisão e classe $)^{10}$, sexo, idade do trabalhador, duração dos benefícios, e a localização geográfica do empregador (unidade federativa).

Este estudo foi realizado com dados do INSS provenientes do Sistema Único de Benefícios (SUB). Foram analisados todos os benefícios auxílio-doença iniciados nos períodos de 01/01/ 2008 a 31/12/2008.

A população de estudo foi definida a partir da média mensal dos registros de emprego declarados em 2008 ao Cadastro Nacional de Informações Sociais (CNIS), também sob administração da Previdência Social, alimentado mensal e eletronicamente, por força de lei, pelas empresas por intermédio das Guias de Recolhimento do Fundo de Garantia por Tempo de Serviço e Informação à Previdência Social (GFIP) ${ }^{1}$.

Os benefícios auxílio-doença do INSS são concedidos a título de compensação salarial quando da incapacidade temporária superior a 15 dias consecutivos, decorrente de qualquer situação que 
se enquadre na definição e equiparação legal de incapacidade para o trabalho. Essa compensação salarial é denominada auxílio-doença que pode ser previdenciário (B31), que é aquele concedido ao segurado impedido de trabalhar por doença ou acidente de natureza não ocupacional; e acidentário (B91), o qual é concedido quando o segurado está incapacitado para o trabalho em decorrência de acidente ou doença do trabalho.

As distorções geradas por valores numéricos (n) muito pequenos, tanto para o CNIS (denominador) quanto para os benefícios (numerador), representam um viés de cálculo importante. Antes de fazer o ranking das prevalências foram separados da análise individual os CNAE com CNIS menor que 500 trabalhadores e/ou menos de 20 benefícios, critérios estes independentes, definidos como parâmetros de corte. Estes dados foram agrupados na categoria Outros, consolidando o total de benefícios. Os resultados agrupados em Outros não foram considerados na análise individualizada das categorias.

Esses dados foram tabulados e analisados no programa Microsoft Office Excel ${ }^{\circledR}$ e no IBM SPSS Statistics Standart versão $19^{\circledR}$. A taxa de prevalência (por 10.000 trabalhadores) foi calculada pela soma dos benefícios auxílio-doença, dividida pela média mensal dos vínculos empregatícios em 2008
A prevalência de benefícios foi calculada e estratificada por: sexo - masculino e feminino; idade - faixas etárias $<20 ; 20-29 ; 30-39 ; 40-49 ; 50$ 59; $\geq 60$ anos e idade dicotomizada em adultos jovens $<40$ anos e adultos $\geq 40$ anos; diagnóstico - capítulo, grupo, e categoria (três dígitos) da CID-10; atividade econômica - CNAE divisão; espécie de benefício auxílio-doença - previdenciário (B31) e acidentário (B91); e por duração do benefício: < 30, 30-59, 60-89, 90-119, 120-149, $150-179,180-269, \geq 270$ dias. Quando mostrados, os intervalos de confiança de $99 \%$ foram calculados utilizando a equação clássica, ponderada por 10.000 , de acordo com a forma de apresentação das prevalências.

Além da análise de prevalência descrita nos parágrafos anteriores, a análise da duração dos benefícios auxílio-doença foi realizada utilizando metodologia não paramétrica, já que a distribuição da duração não apresenta distribuição normal. Para evidenciar diferenças entre grupos, foi utilizada a mediana e para os cálculos de diferenças estatísticas entre grupos (sexo, espécie de benefício e idade dicotomizada) foi utilizado o estimador de Kaplan-Meier. A duração dos benefícios auxílio-doença foi representada graficamente por meio de curva de sobrevivência (Figura 1$)$.

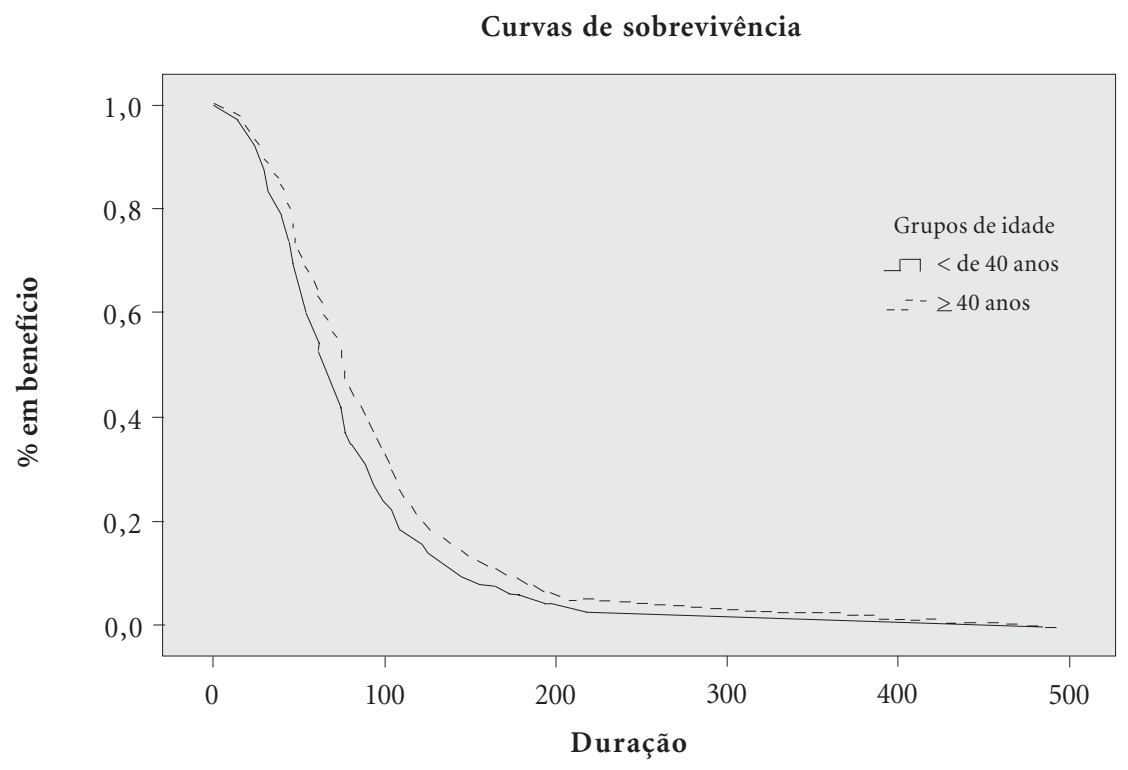

Figura 1. Curvas de sobrevivência da duração dos benefícios auxílio-doença segundo idade dicotomizada, Rondônia - 2008. 


\section{Aspectos éticos}

Este projeto de pesquisa foi submetido e aprovado pelo Comitê de Ética em Pesquisa da Faculdade São Lucas, na cidade de Porto Velho no estado de Rondônia, em 23 de novembro de 2011.

\section{Resultados}

No ano de 2008 o INSS concedeu aos trabalhadores do estado de Rondônia 4.693 benefícios auxílio-doença, dos quais 3.352 foram de natureza previdenciária e 1.341 acidentária; 3.137 masculinos e 556 femininos, 2.986 são adultos jovens ( $<40$ anos) e 1.077 adultos.

A taxa de prevalência de benefícios auxíliodoença em Rondônia no ano de 2008 foi de 262,7 por 10.000 trabalhadores,

Quando da análise das prevalências de benefícios segundo os ramos de atividade econômica, observou-se elevadas taxas na silvicultura e exploração florestal $(1.043,9)$, construção de edifícios $(884,9)$, obras de infraestrutura $(726,1)$, locação de mão de obra $(718,2)$ e fabricação de produtos de metal $(641,8)$ (Tabela 1$)$.
Ao analisar a influência de fatores intrínsecos como sexo e idade na prevalência de incapacidade temporária observou-se que em Rondônia o sexo masculino possui maior prevalência de benefícios auxílio-doença $(\mathrm{RP}=290,0 / 239,1=1,2)$. A maioria das atividades econômicas apresenta o dobro de benefícios para o sexo masculino em relação ao feminino. Todos os benefícios auxíliodoença aumentam com a idade independente do sexo (Tabela 2).

Na população mais jovem, abaixo de 40 anos, a prevalência de benefícios auxílio-doença apresentou uma razão de 1,7 ( $\mathrm{RP}=167,1 / 95,5)$ quando comparado ao grupo mais velho. $\mathrm{Na}$ atividade de fabricação de metais esta diferença foi muito expressiva com $\mathrm{RP}=8,5$, que expressa uma probabilidade superior a oito vezes de trabalhadores com menos de 40 anos apresentarem benefícios auxílio-doença do que os trabalhadores mais velhos (> 40 anos).

Os benefícios auxílio-doença previdenciários são duas vezes e meia mais prevalentes que os acidentários, e também são mais prevalentes em todas as faixas etárias ( $\mathrm{RP}=2,5)$ (Tabela 3 ).

Entre os benefícios previdenciários a diferenca entre os sexos foi de apenas 2\% $(P R=195,2 /$

Tabela 1. Prevalência* de benefícios auxílios-doença segundo atividade econômica CNAE, espécie de benefício e sexo, Rondônia - 2008.

\begin{tabular}{|c|c|c|c|c|c|c|c|c|c|c|c|c|c|c|}
\hline \multirow{2}{*}{ CNAE } & \multicolumn{4}{|c|}{ Benefício previdenciário } & \multicolumn{4}{|c|}{ Benefício acidentário } & \multicolumn{4}{|c|}{ Sexo } & \multirow{2}{*}{ Total } & \multirow[b]{2}{*}{ \pm} \\
\hline & Masc & Fem & Total & \pm & Masc & Fem & Total & \pm & Masc & \pm & Fem & \pm & & \\
\hline 02 & & & & & & & & & & 649,1 & & 1266,6 & 1043,9 & 500 \\
\hline 41 & & 33,9 & & & & a & & & & & & & & 184,9 \\
\hline 42 & 7,7 & 39,4 & 452,8 & 149,9 & 2,6 & 0,0 & 273,3 & 7,5 & & 214,9 & & & 726,1 & 187, \\
\hline 78 & 2,8 & 126,7 & & 309 & & 0,0 & 16 & & & 625,1 & & & & 350,1 \\
\hline 25 & 9,1 & 644,3 & 422,2 & 213,2 & 8 & 61,1 & 219,6 & & & 279,3 & 84,4 & 89 & & 259, \\
\hline 16 & 5,9 & 316,8 & & & 260,0 & 144,8 & & & & 74,8 & 62 & & & 66 , \\
\hline 31 & 8,5 & 292,7 & 0 & 161,5 & 217,0 & 73,2 & 19 & 3 & & 223,2 & 53,2 & &, 5 & 195,8 \\
\hline 43 & 404,1 & 228,6 & 373,5 & 216,9 & 202,1 & 0,0 & 176,9 & 15 & & 291,7 & 19 & &, 4 & 260,9 \\
\hline 01 & 456,3 & 395,2 & 437,1 & 208,4 & 91,3 & 0,0 & 78,1 & 89,7 & & 250,7 & 46,8 & 576,9 & 15,2 & 225,3 \\
\hline 10 & 306,8 & 294,5 & 297,6 & & 237,9 & 157,5 & 212,2 & 35,3 & & 65,5 & 118,7 & & & 53 \\
\hline 49 & & & & & & & & & & & & & & \\
\hline 23 & 201,0 & 460,5 & 218,2 & 76,5 & & 92,1 & 140,0 & 61,5 & & 102,6 & 49,4 & 400,0 & 58,2 & 97 \\
\hline 15 & 206,4 & 169,7 & 193,2 & 119,7 & & 226,2 & 159,1 & 108,9 & 272,8 & 183,0 & 79,6 & 378,3 & 352,4 & 160 , \\
\hline 11 & 232,4 & 112,0 & 208,2 & 132,9 & 139,4 & 112,0 & 130,1 & 105,5 & 312,3 & 192,2 & 26,0 & 404,2 & 338,3 & 168, \\
\hline 56 & 237,1 & 221,4 & 214,8 & 66,0 & 127,7 & 82,4 & 93,4 & 43,8 & 124,5 & 146,1 & 183,6 & 100,5 & 308,1 & 78 , \\
\hline Outros & 145,5 & 178,8 & 159,4 & 8,8 & 48,5 & 38,7 & 44,4 & 4,6 & 194,0 & 12,6 & 217,5 & 15,8 & 197,5 & 9 , \\
\hline Total & 195,2 & 190,7 & 193,5 & 7,3 & 94,9 & 48,4 & 77,4 & 4,3 & 290,0 & 8,0 & 239,1 & 5,7 & 262,7 & \\
\hline \pm & 10,9 & 13,8 & 8,3 & 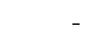 & 7,6 & 7,0 & 5,3 & - & 8,0 & 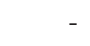 & 5,7 & 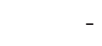 & 9,8 & \\
\hline
\end{tabular}

$\left.{ }^{*}\right)$ por 10.000 trabalhadores. $( \pm)$ intervalo de confiança de 99\%. CNAE: 01 Agricultura, Pecuária e Serviços Relacionados; 02 Silvicultura e Exploração Florestal; 10 Fabricação de Produtos Alimentícios; 11 Fabricação de Bebidas; 15 Preparação de Couros e Fabricação de Artefatos de Couro, Artigos para Viagem e Calçados; 16 Fabricação de Produtos de Madeira; 23 Fabricação de Produtos de Minerais Não Metálicos; 25 Fabricação de Produtos de Metal, exceto Máquinas e Equipamentos; 31 Fabricação de Móveis; 41 Construção de Edifícios; 42 Obras Infraestrutura; 43 Serviços Especializados para Construção, 49 Transporte Terrestre; 56 Alimentação; 78 Seleção, Agenciamento e Locação de mão de obra. 
Tabela 2. Prevalência* de benefícios auxílios-doença segundo atividade econômica CNAE, sexo e faixa de duração*, Rondônia - 2008.

\begin{tabular}{|c|c|c|c|c|c|c|c|c|c|c|c|c|c|c|c|c|c|c|}
\hline \multirow{2}{*}{ CNAE } & \multicolumn{8}{|c|}{ Masculino } & \multicolumn{8}{|c|}{ Feminino } & \multirow[b]{2}{*}{ Total } & \multirow[b]{2}{*}{ \pm} \\
\hline & $\begin{array}{c}< \\
30\end{array}$ & $\begin{array}{c}30- \\
59\end{array}$ & $\begin{array}{c}60- \\
89\end{array}$ & $\begin{array}{l}90- \\
119\end{array}$ & $\begin{array}{c}120- \\
149\end{array}$ & $\begin{array}{c}150- \\
179\end{array}$ & $\begin{array}{c}180- \\
269\end{array}$ & $\begin{array}{c}\geq \\
270\end{array}$ & $\begin{array}{l}< \\
30\end{array}$ & $\begin{array}{c}30- \\
59\end{array}$ & $\begin{array}{c}60- \\
89\end{array}$ & $\begin{array}{l}90- \\
119\end{array}$ & $\begin{array}{c}120- \\
149\end{array}$ & $\begin{array}{c}150- \\
179\end{array}$ & $\begin{array}{c}180- \\
269\end{array}$ & $\begin{array}{c}\geq \\
270\end{array}$ & & \\
\hline 02 & 245,6 & 307,1 & 61,4 & 429,9 & 0,0 & 0,0 & 61,4 & 61,4 & 0,0 & 0,0 & 720,7 & 0,0 & 0,0 & 0,0 & 0,0 & 0,0 & 1043,9 & 556,2 \\
\hline 41 & 70,8 & 251,7 & 228,1 & 180,9 & 118,0 & 78,7 & 31,5 & 70,8 & 140,4 & 140,4 & 46,8 & 0,0 & 46,8 & 0,0 & 0,0 & 0,0 & 884,9 & 184,9 \\
\hline 42 & 27,6 & 258,0 & 175,1 & 184,3 & 46,1 & 27,6 & 64,5 & 36,9 & 0,0 & 119,7 & 0,0 & 59,9 & 59,9 & 0,0 & 0,0 & 0,0 & 726,1 & 187,1 \\
\hline 78 & 158,8 & 158,8 & 264,7 & 423,5 & 105,9 & 105,9 & 0,0 & 52,9 & 0,0 & 0,0 & 63,4 & 0,0 & 63,4 & 0,0 & 0,0 & 0,0 & 718,2 & 350,1 \\
\hline 25 & 58,4 & 272,7 & 136,4 & 77,9 & 39,0 & 0,0 & 58,4 & 0,0 & 161,1 & 161,1 & 161,1 & 0,0 & 161,1 & 0,0 & 161,1 & 0,0 & 641,8 & 259,9 \\
\hline 16 & 72,6 & 180,1 & 146,7 & 100,2 & 55,2 & 21,8 & 24,7 & 14,5 & 81,5 & 0,0 & 0,0 & 0,0 & 0,0 & 0,0 & 9,1 & 0,0 & 582,4 & 66,9 \\
\hline 31 & 51,1 & 217,0 & 140,4 & 102,1 & 51,1 & 12,8 & 38,3 & 12,8 & 73,2 & 73,2 & 146,3 & 0,0 & 73,2 & 0,0 & 0,0 & 0,0 & 574,5 & 195,8 \\
\hline 43 & 44,9 & 112,3 & 202,1 & 89,8 & 89,8 & 22,5 & 44,9 & 0,0 & 228,6 & 0,0 & 0,0 & 0,0 & 0,0 & 0,0 & 0,0 & 0,0 & 550,4 & 260,9 \\
\hline 01 & 54,8 & 127,8 & 127,8 & 54,8 & 73,0 & 91,3 & 0,0 & 18,3 & 131,7 & 131,7 & 0,0 & 0,0 & 0,0 & 0,0 & 0,0 & 131,7 & 515,2 & 225,3 \\
\hline 10 & 71,4 & 166,6 & 152,8 & 81,4 & 32,6 & 17,5 & 13,8 & 8,8 & 51,4 & 0,0 & 0,0 & 0,0 & 0,0 & 0,0 & 20,5 & 6,8 & 509,8 & 53,8 \\
\hline 49 & 27,2 & 126,7 & 65,6 & 49,8 & 56,6 & 20,4 & 20,4 & 27,2 & 72,5 & 58,0 & 58,0 & 43,5 & 58,0 & 0,0 & 29,0 & 0 & 375,4 & 67,9 \\
\hline 23 & 42,1 & 112,2 & 60,8 & 46,7 & 23,4 & 18,7 & 32,7 & 14,0 & 46,0 & 0,0 & 0,0 & 0,0 & 0,0 & 0,0 & 0,0 & 0,0 & 358,2 & 97,3 \\
\hline 15 & 29,5 & 103,2 & 147,5 & 29,5 & 0,0 & 14,7 & 14,7 & 14,7 & 0,0 & 0,0 & 0,0 & 0,0 & 0,0 & 0,0 & 0,0 & 56,6 & 352,4 & 160,4 \\
\hline 11 & 62,0 & 124,0 & 77,5 & 31,0 & 31,0 & 15,5 & 15,5 & 15,5 & 0,0 & 0,0 & 0,0 & 0,0 & 0,0 & 0,0 & 0,0 & 0,0 & 338,3 & 168,3 \\
\hline 56 & 45,6 & 91,2 & 109,4 & 36,5 & 18,2 & 27,4 & 27,4 & 9,1 & 30,9 & 92,7 & 66,9 & 82,4 & 15,4 & 5,1 & 5,1 & 5,1 & 308,1 & 78,7 \\
\hline Outros & 112,4 & 343,1 & 299,9 & 188,3 & 70,0 & 51,3 & 64,7 & 29,8 & 926,5 & 1837,0 & 1598,8 & 1000,6 & 402,3 & 211,8 & 291,2 & 137,6 & 486,4 & 7,6 \\
\hline Total & 43,4 & 127,9 & 109,0 & 69,7 & 29,6 & 19,1 & 22,2 & 12,3 & 60,2 & 111,1 & 96,4 & 61,2 & 25,2 & 12,4 & 18,4 & 8,8 & 262,7 & 12,2 \\
\hline \pm & 3,1 & 5,3 & 4,9 & 4,0 & 2,6 & 2,1 & 2,2 & 1,7 & 2,9 & 3,9 & 3,6 & 2,9 & 1,8 & 1,3 & 1,6 & 1,1 & 12,2 & . \\
\hline
\end{tabular}

(") por 10.000 trabalhadores. (*) em dias. ( \pm ) intervalo de confiança de 99\%. CNAE: 01 Agricultura, Pecuária e Serviços Relacionados; 02 Produção Florestal; 10 Fabricação de Produtos Alimentícios; 11 Fabricação de Bebidas; 15 Preparação de Couros e Fabricação de Artefatos de Couro, Artigos para Viagem e Calçados; 16 Fabricação de Produtos de Madeira; 23 Fabricação de Produtos de Minerais Não Metálicos; 25 Fabricação de Produtos de Metal, exceto Máquinas e Equipamentos; 31 Fabricação de Móveis; 42 Construção de Edifícios; 43 Serviços Especializados para Construção, 49 Transporte Terrestre; 56 Alimentação; 78 Seleção, Agenciamento e Locação de mão de obra.

Tabela 3. Prevalência* de benefícios auxílios-doença segundo capítulos de CID-10, espécie de benefício e sexo, Rondônia - 2008.

\begin{tabular}{|c|c|c|c|c|c|c|c|c|c|c|}
\hline \multirow[b]{2}{*}{ Capítulo CID } & \multicolumn{4}{|c|}{ Benefício previdenciário } & \multicolumn{4}{|c|}{ Benefício acidentário } & \multirow[b]{2}{*}{ Total } & \multirow[b]{2}{*}{ \pm} \\
\hline & Masc & Fem & Total & \pm & Masc & Fem & Total & \pm & & \\
\hline XIX & 74,8 & 33,7 & 59,3 & 4,6 & 64,1 & 17,5 & 46,6 & 4,1 & 102,7 & 6,2 \\
\hline XIII & 38,2 & 46,7 & 41,4 & 3,9 & 25,4 & 25,0 & 25,3 & 3,0 & 64,6 & 4,9 \\
\hline XI & 21,3 & 11,7 & 17,7 & 2,5 & 1,2 & 0,3 & 0,9 & 0,6 & 18,0 & 2,6 \\
\hline IX & 15,1 & 14,8 & 15,0 & 2,3 & 0,5 & 0,8 & 0,6 & 0,5 & 15,1 & 2,4 \\
\hline $\mathrm{V}$ & 9,3 & 14,8 & 11,4 & 2,0 & 0,5 & 1,5 & 0,9 & 0,6 & 11,9 & 2,1 \\
\hline XIV & 4,6 & 17,2 & 9,4 & 1,8 & 0,0 & 0,0 & 0,0 & 0,0 & 9,1 & 1,8 \\
\hline I & 9,0 & 4,1 & 7,2 & 1,6 & 1,2 & 0,8 & 1,0 & 0,6 & 7,9 & 1,7 \\
\hline II & 3,5 & 14,8 & 7,7 & 1,7 & 0,0 & 0,0 & 0,0 & 0,0 & 7,5 & 1,7 \\
\hline XV & 0,0 & 19,5 & 7,3 & 1,6 & 0,0 & 0,0 & 0,0 & 0,0 & 7,1 & 1,6 \\
\hline VI & 5,1 & 3,5 & 4,5 & 1,3 & 0,3 & 1,5 & 0,8 & 0,5 & 5,1 & 1,4 \\
\hline VII & 5,5 & 2,6 & 4,4 & 1,3 & 0,8 & 0,2 & 0,6 & 0,5 & 4,8 & 1,3 \\
\hline Outros & 8,9 & 7,4 & 8,3 & 1,7 & 0,9 & 0,8 & 0,9 & 0,6 & 8,9 & 1,8 \\
\hline Total & 195,2 & 190,7 & 193,5 & 8,3 & 94,9 & 48,4 & 77,4 & 5,3 & 262,7 & 9,8 \\
\hline \pm & 10,9 & 13,8 & 8,3 & - & 7,6 & 7,0 & 5,3 & - & 9,8 & - \\
\hline
\end{tabular}

$\left({ }^{*}\right)$ por 10.000 trabalhadores. ( \pm ) intervalo de confiança de 99\%. Capítulos CID-10 - Descrição: I - Algumas doenças infecciosas e parasitárias; II - Neoplasias (tumores); V - Transtornos mentais e comportamentais; VI - Doenças do sistema nervoso; VII Doenças do olho e anexos; VIII - Doenças do ouvido e da apófise mastóide; IX - Doenças do aparelho circulatório; X - Doenças do aparelho respiratório; XI - Doenças do aparelho digestivo; XIII - Doenças sistema osteomuscular e tecido conjuntivo; XIV Doenças do aparelho geniturinário; XV - Gravidez, parto e puerpério; XIX - Lesões, envenamento e algumas outras consequências causas externas; XXI - Fatores que influenciam o estado de saúde e o contato com serviços de saúde. 
190,7 = 1,0), contrapondo-se aos acidentários para os quais a razão de masculinidade foi de 1,94 , mostrando-se uma probabilidade de benefícios acidentários de quase o dobro do que o sexo feminino. No conjunto dos benefícios auxílio-doença, as maiores prevalências foram observadas nos grupos das lesões, envenenamento e algumas outras consequências de causas externas (lesões), das doenças do sistema osteomuscular e tecido conjuntivo (doenças osteomusculares) e das doenças do aparelho digestivo.

Os grupos-CID mais prevalentes foram: dorsopatias $(37,7)$, traumatismo de joelho e perna $(23,5)$; traumatismo de punho e mão $(22,8)$ e transtornos dos tecidos moles e músculos $(17,0)$.

Entre os benefícios previdenciários os grupos-CID mais prevalentes foram das doenças do aparelho digestivo; doenças do aparelho circulatório e transtornos mentais e comportamentais; e entre os acidentários predominaram as lesões, as doenças osteomusculares e as doenças do aparelho digestivo. As maiores prevalências de benefícios acidentários segundo a categoria diagnóstica apontam traumatismos do punho e da mão $(15,9)$ e de transtornos dos tecidos moles e músculos $(10,6)$ como os mais prevalentes (Tabela 4).

As maiores prevalências de benefícios auxílio-doença ocorreram em decorrência de lesões e doenças osteomusculares e nas faixas etárias mais altas (40-49; 60-69). As lesões são mais prevalentes no sexo masculino do que no feminino, em especial nas faixas etárias dos 20-29 e 30-39 anos. Entre o sexo feminino observa-se uma prevalência máxima aos 50-59 anos.

As doenças osteomusculares aumentam com a idade em ambos os sexos, exceto nas mulheres acima de 60 anos quando ocorre um decréscimo. A prevalência de benefícios por dorsopatias foi de 38,4 , enquanto os traumatismos de membros superiores distribuiram-se principalmente no punho e na mão $(23,6)$, no ombro e braço $(11,5)$ e no cotovelo e antebraço $(11,2)$ somam uma prevalência de 46,3. Entre os traumatismos de membros inferiores apresentam prevalência de 36,6 , predominaram os do joelho e da perna $(24,2)$ e do tornozelo e do pé $(12,4)$.

As dorsopatias são duas vezes mais prevalentes entre benefícios previdenciários, com razão de prevalência de 2,0 $(\mathrm{RP}=26,0 / 12,8)$. Estes agravos têm sua prevalência aumentada a partir dos 50 anos de idade em ambos os sexos. As dorsopatias masculinas aumentam com a idade.

O sexo feminino apresenta, em relação ao sexo oposto, elevada prevalência de benefícios devido à dorsopatias $(35,0)$ e transtornos de tecidos moles e músculos $(28,4)$, definidas como doenças musculoesqueléticas, com predominância acima de 40 anos.

As artropatias, doenças do aparelho digestivo e doenças do aparelho circulatório têm maior prevalência nos benefícios previdenciários, mas pouca relevância em benefícios acidentários. Os ho-

Tabela 4. Prevalência* de benefícios auxílios-doença segundo grupo de CID-10, espécie de benefício e faixa etária, Rondônia 2008.

\begin{tabular}{|c|c|c|c|c|c|c|c|c|c|c|c|c|c|c|c|c|c|c|}
\hline \multirow[b]{2}{*}{$\begin{array}{c}\text { Grupo } \\
\text { CID }\end{array}$} & \multicolumn{8}{|c|}{ Benefício previdenciário } & \multicolumn{8}{|c|}{ Benefício acidentário } & \multirow[b]{2}{*}{ Total } & \multirow[b]{2}{*}{ \pm} \\
\hline & $\begin{array}{c}< \\
20\end{array}$ & $\begin{array}{c}20- \\
29\end{array}$ & $\begin{array}{c}30- \\
39\end{array}$ & $\begin{array}{c}40- \\
49\end{array}$ & $\begin{array}{c}50- \\
59\end{array}$ & $\begin{array}{l}\geq \\
60\end{array}$ & Total & \pm & $\begin{array}{c}< \\
20\end{array}$ & $\begin{array}{c}20- \\
29\end{array}$ & $\begin{array}{c}30- \\
39\end{array}$ & $\begin{array}{c}40- \\
49\end{array}$ & $\begin{array}{c}50- \\
59\end{array}$ & $\begin{array}{l}\geq \\
60\end{array}$ & Total & \pm & & \\
\hline M40-M54 & 2,8 & 7,8 & 26,9 & 53,9 & 78,0 & 95,9 & 26,0 & 3,2 & 1,8 & 7,0 & 15,8 & 17,8 & 32,8 & 19,2 & 12,8 & 2,2 & 37,7 & 3,7 \\
\hline S80-S89 & 12,9 & 16,4 & 16,0 & 17,8 & 10,6 & 24,0 & 16,0 & 2,5 & 4,6 & 8,5 & 7,7 & 9,1 & 8,7 & 9,6 & 8,1 & 1,8 & 23,5 & 3,0 \\
\hline S60-S69 & 5,5 & 9,1 & 8,7 & 4,5 & 4,8 & 4,8 & 7,7 & 1,7 & 15,7 & 19,8 & 12,4 & 13,6 & 15,4 & 4,8 & 15,9 & 2,5 & 22,8 & 2,9 \\
\hline M60-M79 & 0,9 & 4,0 & 8,1 & 12,6 & 9,6 & 14,4 & 6,9 & 1,6 & 0,0 & 6,5 & 12,8 & 19,6 & 14,4 & 9,6 & 10,6 & 2,0 & 17,0 & 2,5 \\
\hline S90-S99 & 3,7 & 9,1 & 5,8 & 7,0 & 6,7 & 0,0 & 7,2 & 1,7 & 5,5 & 5,0 & 5,2 & 5,9 & 5,8 & 0,0 & 5,3 & 1,4 & 12,0 & 2,1 \\
\hline $\mathrm{S} 40-\mathrm{S} 49$ & 5,5 & 9,6 & 6,4 & 6,6 & 6,7 & 9,6 & 7,7 & 1,7 & 1,8 & 5,3 & 2,9 & 2,1 & 3,9 & 9,6 & 3,8 & 1,2 & 11,2 & 2,0 \\
\hline S50-S59 & 8,3 & 7,2 & 5,2 & 7,0 & 7,7 & 4,8 & 6,6 & 1,6 & 4,6 & 4,9 & 4,4 & 4,2 & 4,8 & 0,0 & 4,6 & 1,3 & 10,9 & 2,0 \\
\hline M00-M25 & 0,9 & 3,6 & 7,1 & 12,2 & 28,9 & 19,2 & 7,6 & 1,7 & 0,0 & 1,3 & 1,2 & 2,1 & 3,9 & 14,4 & 1,6 & 0,8 & 9,0 & 1,8 \\
\hline K40-K46 & 0,0 & 6,2 & 5,6 & 11,9 & 19,3 & 33,6 & 7,7 & 1,7 & 0,0 & 0,9 & 0,6 & 1,7 & 0,0 & 0,0 & 0,8 & 0,6 & 8,2 & 1,8 \\
\hline I80-I89 & 0,0 & 3,0 & 5,2 & 12,2 & 12,5 & 9,6 & 5,7 & 1,5 & 0,0 & 0,1 & 0,4 & 1,0 & 1,0 & 0,0 & 0,4 & 0,4 & 5,9 & 1,5 \\
\hline Outros & 22,2 & 71,0 & 91,2 & 127,0 & 202,3 & 326,2 & 94,3 & 6,0 & 3,7 & 11,4 & 13,7 & 15,4 & 30,8 & 19,2 & 13,5 & 2,3 & 104,5 & 6,2 \\
\hline Total & 62,8 & 146,9 & 186,3 & 272,8 & 387,2 & 542,0 & 193,5 & 8,5 & 37,9 & 70,7 & 77,1 & 92,7 & 121,4 & 86,3 & 77,4 & 5,4 & 262,7 & 9,8 \\
\hline \pm & 19,6 & 11,8 & 15,3 & 24,9 & 48,9 & 127,9 & 8,5 & - & 15,2 & 8,2 & 9,9 & 14,6 & 27,7 & 52,3 & 5,4 & - & 9,8 & 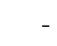 \\
\hline
\end{tabular}

(*) por 10.000 trabalhadores. ( \pm ) intervalo de confiança de 99\%. Grupos CID-10: M40-M54 Dorsopatia; S80-S89 Traumatismo do joelho e da perna; S60-S69 Traumatismo do punho e da mão; M60-M79 Trauma de partes moles e músculos, S90-S99 Traumatismo do tornozelo e do pé; S40-S49 Traumatismos do ombro e do braço; S50-S59 Traumatismos do cotovelo e do antebraço; M00-M25 Artropatias; K40-K46 Hérnias; I80-I89 Doenças das veias. 
mens tiveram maior prevalência de doenças do aparelho digestivo - em especial hérnias $(\mathrm{RP}=$ $6,9)$ e artropatias $(\mathrm{RP}=1,4)$. As mulheres tiveram maior prevalência de doenças do aparelho circulatório $(\mathrm{RP}=1,3)$. As doenças do aparelho digestivo aumentam independente da faixa etária no sexo masculino enquanto que no feminino isto ocorre aos 40-49 anos, já as doenças do aparelho circulatório crescem com a faixa etária em ambos os gêneros.

Os benefícios acidentários decorrentes de transtornos mentais são mais prevalentes no sexo feminino e os decorrentes de neoplasias (tumores) são insignificantes ou nulos.

Os traumatismos do joelho e da perna apresentaram o dobro de prevalência de benefícios auxílio-doença previdenciários em relação aos benefícios auxílio-doença acidentários com razão de prevalência aproximada de 2,0.

Os traumatismos do punho e da mão são mais prevalentes em homens e na faixa etária de 20-29 anos $(43,8)$ e entre benefícios acidentários. Além dos traumatismos do punho e da mão, o único agrupamento clínico com predominância de benefícios acidentários foram os transtornos de partes moles e músculos.

Observou-se maior prevalência de traumas do joelho e da perna, com forte caracterização previdenciária $(\mathrm{RP}=2)$.

$\mathrm{Na}$ atividade econômica de Carne e Pescado em Rondônia, os traumatismos de membros superiores e inferiores apresentaram as maiores prevalências de benefícios. Os traumatismos de membros superiores são mais prevalentes.

As maiores prevalências de benefícios acidentários decorrentes de lesões e doenças osteomusculares ocorreram nas atividades econômicas silvicultura e exploração florestal; construção de edifícios e obras de infraestrutura (construção civil).

Os benefícios acidentários decorrentes de lesões apresentam expressiva prevalência na silvicultura e exploração florestal; fabricação de produtos de madeira; e fabricação de produtos de metal, exceto máquinas e equipamentos. Os decorrentes de doenças osteomusculares apresentam maiores prevalências nas atividades preparação de couros e fabricação de artefatos de couro, artigos para viagem e calçados; seleção e gerenciamento e locação de mão de obra; e atividades financeiras, de seguros e serviços relacionados.

Em Rondônia a maior duração dos benefícios ocorre na faixa de 30-59 dias de afastamento, seguida da faixa de 60-89 dias e declínio acentuado ate os 120-149 dias, em ambos os sexos ( Tabela 2).
Os benefícios auxílios-doença concedidos ao sexo feminino foram mais curtos do que ao sexo masculino $(p<0,001)$, com medianas cinco dias inferior.

A duração dos benefícios concedidos aos trabalhadores adultos ( $\geq 40$ anos de idade) é maior do que a duração dos benefícios concedidos aos adultos jovens ( $<40$ anos), com diferença da mediana de onze dias superior $(p<0,0001)$ (Figura 1).

A duração dos benefícios foi maior entre os previdenciários comparados aos acidentários, com mediana sete dias superior. A diferença entre as espécies de benefícios independem do sexo ou do CNAE $(p<0,001)$. Entre a espécie de beneficio previdenciário, os afastamentos mais longos ocorreram nas atividades econômicas de agricultura e pecuária e serviços relacionados; construção civil (representada pelos ramos da construção de edifícios; obras infraestrutura; e serviços especializados para construção), seguidos pela fabricação de produtos da madeira.

Nos benefícios acidentários a duração foi mais expressiva nas atividades econômicas de construção civil representada pelos subramos da construção de edifícios; obras infraestrutura e serviços especializados para construção, e em especial até a faixa de 120-149 dias de afastamento do trabalho (dados não tabulados).

As atividades econômicas com duração de afastamentos do trabalho superiores a seis meses foram a construção civil; a fabricação de produtos de metal; a agricultura e pecuária; e a silvicultura e exploração florestal.

Em 2008, o INSS de Rondônia concedeu 1954 benefícios auxílio-doença devido a lesões. Esses benefícios, segundo o sexo foram distribuídos a 1.609 homens e 345 mulheres. Os benefícios previdenciários apresentaram maior duração que os acidentários devido a lesões em Rondônia ( $p<$ $0,001)$. Segundo a idade dicotomizada, os adultos jovens $(<40$ anos $)$ obtiveram uma RP = $1.513 / 441=3,4(p<0,001)$. Os trabalhadores adultos ( $\geq 40$ anos) obtiveram benefícios mais duradouros em 7 dias na mediana, com forte significância estatística. $(p<0,001)$. Em relação à mediana da duração dos benefícios auxílio-doença decorrentes de lesões no Brasil, que foi calculada em 60 dias, os benefícios concedidos em Rondônia foram 10 dias mais duradouros. Os benefícios previdenciários em Rondônia tiveram duração de 16 dias acima da mediana dos trabalhadores brasileiros, enquanto entre os acidentários, essa diferença foi de apenas dois dias (dados não tabulados). 


\section{Discussão}

A taxa de prevalência de benefícios auxílio-doença concedidos pelo INSS em Rondônia no ano de 2008 foi de 262,7 por 10 mil, a qual é considerada baixa quando comparada à média nacional, para o mesmo ano, de 421,8 por 10 mil $^{1}$ e às taxas europeias $1.050,0^{11}$ e $1.370,0$ por $10 \mathrm{mil}^{12}$.

A baixa demanda por benefícios auxílio-doença, por parte do trabalhador empregado rondoniense, pode estar associada a uma menor conscientização dos direitos dos trabalhadores, menor nível de escolaridade, pela sindicalização precária, bem como pela dificuldade de acesso aos postos da previdência, em sua maioria restrita a médios e grandes centros urbanos.

Em Rondônia o sexo masculino possui maior prevalência de benefícios auxílio-doença ao contrário do observado em estudo nacional de Barbosa-Branco et al. ${ }^{1}$. A maioria das atividades econômicas apresenta o dobro de benefícios para o sexo masculino em relação ao feminino.

A elevada prevalência de benefícios concedidos aos ramos agrosilvopastoril é corroborada por estudo anterior sobre os riscos ocupacionais em Rondônia, o qual mostrou predominância de trabalhadores rurais e braçais a empresas de pequeno porte ( $<10$ empregados). Essas empresas atuam no agronegócio e na prestação de serviços, com uma industrialização rudimentar gerando características de riscos ocupacionais distintos de grande parte dos demais estados brasileiros que possuem maior industrialização e desenvolvimento socioeconômico ${ }^{1,8,11-14}$.

A elevada prevalência de beneficios auxíliodoença entre os trabalhadores da atividade econômica de seleção, agenciamento e locação de mão de obra aponta para a importância do processo de terceirização tanto da mão de obra quanto de certos processos produtivos. Este processo de terceirização agrega a ele a transferência de riscos à saúde dos trabalhadores, bem como, contribui para a subnotificação dos agravos.

Todos os benefícios auxílio-doença aumentam com a idade independente do sexo, resultado semelhante aos estudos de Barbosa-Branco ${ }^{1}$, Roelen et al. ${ }^{11}$, Elders' et al. ${ }^{15}$ e Kines et al. ${ }^{16}$.

Nos benefícios previdenciários os grupos-CID mais prevalentes doenças do aparelho digestivo; doenças do aparelho circulatório e transtornos mentais e comportamentais; e entre os acidentários as lesões, as doenças osteomusculares e as doenças do aparelho digestivo corroboram com os estudos brasileiros de Almeida ${ }^{17}$ e Barbosa-Branco et al. ${ }^{1}$.
As doenças osteomusculares aumentam com a idade em ambos os sexos, exceto nas mulheres acima de 60 anos quando ocorre um decréscimo. Este provavelmente ocorra devido à aposentadoria por idade, que no sexo feminino e de 60 anos. A idade é forte fator de risco para doenças musculoesqueléticas ${ }^{16}$.

As artropatias, doenças do aparelho digestivo e doenças do aparelho circulatório têm maior prevalência nos benefícios previdenciários, mas pouca relevância em benefícios acidentários. Os homens tiveram maior prevalência de doenças do aparelho digestivo - em especial hérnias ( $\mathrm{RP}=$ $6,9)$ e artropatias ( $\mathrm{RP}=1,4)$. As mulheres tiveram maior prevalência de doenças do aparelho circulatório $(\mathrm{RP}=1,4)$. Estes dados corroboram com os autores Ashbury ${ }^{18}$, Meservy et al. ${ }^{19}$, Gluck $e$ Oleinick ${ }^{20}$, Lindqvist et al. ${ }^{21}$ e Islan et al. ${ }^{22}$.

Os transtornos mentais são mais prevalentes no sexo feminino e agora começam a aparecer entre os benefícios acidentários devido à implantação do Nexo Técnico Epidemiológico Previdenciário (NTEP) pelo INSS.

Os benefícios acidentários decorrentes de neoplasias (tumores) são insignificantes ou nulos. Corrêa $^{23}$ infere a existência de um verdadeiro silêncio epidemiológico no câncer relacionado ao trabalho no Brasil. A invisibilidade do câncer relacionado ao trabalho pode ser avaliada pela irrisória participação das neoplasias relacionadas ao trabalho dentre os auxílios-doença acidentários concedidos pela Previdência Social. O lapso de tempo e o desconhecimento do assunto dificultam o nexo causal e o seu registro adequado no sistema de notificação ${ }^{24}$.

Os transtornos mentais e as neoplasias e algumas outras enfermidades apresentam dificuldade no reconhecimento do nexo causal pelos médicos, classificando estas enfermidades relacionadas ao trabalho como benefícios previdenciários. Isto contribui substancialmente para o $s u$ bregistro de benefícios acidentários. Isto poderia ajudar a explicar as baixas taxas de doenças relacionadas ao trabalho encontradas no Brasil. Com a implantação do NTEP foi facilitado o trabalho pericial no estabelecimento do nexo técnico epidemiológico por parte dos médicos da PS, definindo as doenças relacionadas ao trabalho como benefícios acidentários.

No Brasil, os traumatismos do punho e da mão são mais prevalentes em relação a traumatismo do joelho e da perna ${ }^{25}$. Em contrapartida, no estado de Rondônia observou-se maior prevalência de traumas do joelho e da perna, com forte caracterização previdenciária $(\mathrm{RP}=2)$. Este fato ocor- 
re provavelmente devido a alta prevalência de acidentes de trânsito, em especial envolvendo as motocicletas, meio de transporte popular, rápido e econômico, mas extremamente inseguro ${ }^{26,27}$.

Lipton et al. ${ }^{28}$ estimaram que a média de duração de benefícios nos Estados Unidos no ano de 2010 foi de 125 dias, enquanto que a mediana americana ficou em 42 dias. Almeida ${ }^{17}$ constatou média de 59 dias em benefícios acidentários no Brasil no ano de 2008. Em Rondônia, o maior número de benefícios apresenta duração entre 30-59 dias de afastamento.

Os benefícios auxílios-doença concedidos ao sexo feminino, mais curtos do que ao sexo masculino $(p<0,001)$, corroboram tanto os dados do estudo de base nacional sobre os benefícios auxílio-doença concedidos pelo INSS no Brasil em $2008^{1}$, quanto o estudo europeu ${ }^{29}$.

A maior duração dos benefícios concedidos aos trabalhadores adultos ( $\geq 40$ anos de idade), com $(p<0,0001)$ reitera outros estudos de Elders et al. ${ }^{15}$, Kines et al. ${ }^{16}$, Roelen et al. ${ }^{11}$ e BarbosaBranco et al. ${ }^{1}$, demonstrando que a idade é forte fator de risco para a incapacidade para o trabalho. Elders et al. ${ }^{15}$ argumentaram que com o avanço da idade aumentam as taxas de doenças relacionados ao trabalho. Possivelmente, ocorra maior dificuldade de recuperação da capacidade laboral decorrente da senilidade, considerando também que a idade possa ser um forte fator de risco para a longa duração dos benefícios (Figura 1$)$.

Outros fatores não estritamente médicos, como posição socioeconômica baixa, idade abaixo de 39 anos, expectativa alta de retorno ao trabalho e reposição de renda pelo INSS menor ou igual a $100 \%$, podem justificar a maior taxa de cessação do benefício e sua menor duração segundo o estudo de coorte realisado com trabalhadores da Bahia ${ }^{30}$.

A maior durabilidade dos benefícios decorrentes de lesões no estado de Rondônia aponta para uma baixa resolutividade dos serviços médico-hospitalares prestados em urgência e emergência, em especial nos serviços públicos do Sistema Único de Saúde (SUS).

Os adultos jovens rondonienses $(<40$ anos) obtiveram uma $\mathrm{RP}=3,43$ de lesões maior que outros estudos brasileiros, $\mathrm{RP}=2,71^{1,31}$, possivelmente reflexo das altas taxas de violência urbana envolvendo jovens no estado.

Interessante observar que existe íntima relação entre as principais atividades econômicas, o contexto do desenvolvimento regional, as maiores taxas de prevalências e a duração de benefíci- os auxílio-doença apuradas neste estudo. A atividade econômica é um importante preditor das condições de trabalho e desempenha um papel relevante na prevalência e nas características das reivindicações previdenciárias tanto no mundo quanto no Brasil ${ }^{32,33}$.

A maior limitação desta pesquisa está relacionada à população de estudo, que não compreende a totalidade da população trabalhadora, na medida em que exclui os trabalhadores autônomos, os servidores públicos e os trabalhadores informais.

Cockell e Perticarrari ${ }^{34}$ inferem que o trabalhador informal, quando sua vida não é perdida no exercício do trabalho, a duração do tratamento e o tempo de recuperação distinguem-se dos casos cobertos pelo INSS. Diante das dificuldades financeiras e da desproteção social, esses trabalhadores antecipam o retorno ao trabalho antes do término do tratamento, caso tenham recebido tal assistência, e mesmo sem o restabelecimento completo de suas forças físicas e mentais, voltam a exercer suas atividades para garantir o sustento familiar.

\section{Conclusões}

As atividades de silvicultura e exploração florestal; a construção civil (construção de edifícios; obras infraestrutura e serviços especializados para construção) e a gestão de mão de obra são as mais vulneráveis em relação à incapacidade para o trabalho.

A atividade econômica de seleção, agenciamento e locação de mão de obra demonstra o grande número de benefícios auxilio-doença nas empresas terceirizadas, alertando para a transferência de funções de risco das grandes para pequenas empresas.

O estado possui predominância de trabalhadores rurais e braçais envolvidos no agronegócio e na prestação de serviços com uma industrialização rudimentar gerando características de riscos ocupacionais distintos de outros estados brasileiros que possuem melhor desenvolvimento socioeconômico.

Este trabalho fornece informações que poderiam ser úteis na implantação de políticas previdenciárias e de saúde do trabalhador a fim de reduzir as incapacidades para o trabalho e as consequências econômicas e sociais dela decorrentes.

Os resultados desta pesquisa podem subsidiar novas explorações mais detalhadas da relação entre a atividade econômica (riscos ocupacionais), a incapacidade para o trabalho e o desen- 
volvimento socioeconômico das diversas regiões brasileiras.

\section{Principais achados}

1. A taxa de prevalência de benefícios auxílio-doença em Rondônia em 2008 foi de 262,7 por 10 mil trabalhadores, com predominância masculina.

2. As atividades de silvicultura e exploração florestal; a construção civil e a gestão de mão de obra são as mais vulneráveis em relação à incapacidade para o trabalho.

3. A atividade de seleção, agenciamento e locação de mão de obra aponta para a importância do processo de terceirização tanto da mão de obra quanto de certos processos produtivos, com transferência de riscos ocupacionais, o que con- tribui para a subnotificação dos agravos à saude do trabalhador.

4. Os benefícios previdenciários mais prevalentes foram as doenças do aparelho digestivo; as doenças do aparelho circulatório e os transtornos mentais e comportamentais; e os acidentários as lesões, as doenças osteomusculares e as doenças do aparelho digestivo.

5. A maior prevalência de traumas do joelho e da perna, em relação aos traumatismos do punho e da mão, com caracterização previdenciária, decorre em grande parte da alta prevalência de acidentes de trânsito, em especial envolvendo as motocicletas.

6. A maior duração dos benefícios decorrentes de lesões aponta para uma baixa resolutividade dos serviços médico-hospitalares prestados em urgência e emergência no estado.

\section{Colaboradores}

HR Jakobi, A Barbosa-Branco, LF Bueno, RGM Ferreira e LMA Camargo participaram igualmente de todas as etapas de elaboração do artigo. 


\section{Referências}

1. Barbosa-Branco A, Souza WR, Steenstra IA. Incidence of work and non-work related benefit claims in Brazil. Am J Ind Med 2011; 54(11):858-871.

2. Mendes R, Dias EC. Saúde dos trabalhadores. In: Rouquayrol MZ, Almeida Filho N, organizadores. Epidemiologia \& Saúde. 5a Edição. Rio de Janeiro: Medsi; 1999. p. 431-456.

3. Viana GA. Relatório do desenvolvimento (in)sustentável da Amazônia: o caso das madeireiras. Brasília: Câmara dos Deputados; 2000.

4. Nascimento EP, Drummond JA. Amazônia, dinamismo econômico e conservação ambiental. Rio de Janeiro: Ed. Garamond; 2003.

5. Oliveira AU. BR-163 Cuiabá-Santarém: geopolítica, grilagem, violência e mundialização. In: Torres M, organizador. Amazônia revelada: os descaminhos ao longo da BR-163. Brasília: CNPq; 2005. p. 67-183.

6. Pignati WA. Riscos e agravos à saúde e à vida dos trabalhadores das indústrias madeireiras de Mato Grosso. Cien Saude Colet 2005; 10(4):961-973.

7. Pignati WA. Os riscos, agravos e vigilância em saúde no espaço de desenvolvimento do agronegócio no Mato Grosso [tese]. Rio de Janeiro: Escola Nacional de Saúde Pública Sergio Arouca; 2007.

8. Jakobi HR. Mapa de Risco Ocupacional no estado de Rondônia baseado em tecnologia de georeferenciamento [dissertação]. Porto Velho: Universidade Federal de Rondônia; 2008.

9. Organização Mundial de Saúde (OMS). Classificação Estatística Internacional de Doenças e Problemas Relacionados à Saúde 10 Revisão (CID-10). 2008. [acessado 2011 abr 12]. Disponível em: http://www. datasus.gov.br/cid10/v2008/cid10.htm

10. Instituto Brasileiro de Geografia E Estatística (IBGE). Cadastro Nacional de Atividades Econômicas - CNAE 2.0. 2008. [acessado 2010 abr 12]. Disponível em: http://www.ibge.gov.br/home/estatistica/ economia/classificacoes/cnae2.0_subclasses/ default.shtm

11. Roelen CAM, Koopmans PC, Hoedeman R, Bültmann U, Groothoff JW, van der Klink JJ. Trends in the incidence of sickness absence due to common mental disorders between 2001 and 2007 in the Netherlands. Eur J Public Health 2009; 19(6):625-630.

12. Ferrie JE, Vahtera J, Kivimäki $M$, Westerlund $H$, Melchior M, Alexanderson K, Head J, Chevalier A, Leclerc A, Zins M, Goldberg M, Singh-Manoux A. Diagnose-specific sickness absence and all cause mortality in the GAZEL study. J Epidemiol Community Health 2009; 63(1):50-55

13. Zwerling C. Occupational injuries - Comparing the rates of male and female postal workers. Am J Epidemiol 1993; 138(1):46-55.

14. Wohl AR, Morgenstern H, Kraus JF. Occupational injury in female aerospace workers. Epidemiol 1995; 6(2):110-114

15. Elders LA, Heinrich J, Burdorf A. Risk factors for sickness absence because of low back pain among scaffolders: a 3-year follow-up study. Spine 2003; 28(12):1340-1346
16. Kines P, Hannerz H, Mikkelsen KL, Tüchsen F. Industrial sectors with high risk of women's hospitaltreated injuries. Am J Ind Med 2007; 50(1):13-21.

17. Almeida PCA. Prevalência, duração e despesa previdenciária da incapacidade temporária por acidentes do trabalho no Brasil [dissertação]. Brasília: Universidade de Brasília; 2011.

18. Ashbury FD. Occupational repetitive strain injuries and gender in Ontario, 1986 to 1991. J Occup Environ Med 1995; 37(4):479-485.

19. Meservy D, Suruda AJ, Bloswick D, Lee J, Dumas M. Ergonomic risk exposure and upper-extremity cumulative trauma disorders in a Maquiladora Medical Devices Manufacturing plant. J Occup Environ Med 1997; 39(8):767-773.

20. Gluck JV, Oleinick A. Claim rates of compensable back injuries by age, gender, occupation, and industry. Spine 1998; 23(14):1572-1586.

21. Lindqvist K, Schelp L, Timpka T. Gender aspects of work-related injuries in a Swedish municipality. Safety Science 1999; 31(3):183-196.

22. Islam SS, Velilla AM, Doyle EJ, Ducatman AM. Gender differences in work-related injury/illness: Analysis of workers compensation claims. Am J Ind Med 2001; 39(1):84-91.

23. Corrêa MJM. A construção social do silêncio epidemiológico do benzenismo: uma historia negada [dissertação]. Porto Alegre: Pontifica Católica do Rio Grande do Sul; 2008.

24. Instituto Nacional do Câncer (INCA). Diretrizes para a vigilância do câncer relacionado ao trabalho. Rio de Janeiro: Inca; 2012.

25. Brasil. Ministério da Previdência Social. Anuário Estatístico da Previdência Social (AEPS) 2008. [acessado 2013 ago 4]. Disponível em: http://www. previdencia.gov.br/arquivos/office/3_091028191015-957.pdf

26. Orsati FT, Machado FS, Kitayama NM, Bruscato WL. Estudo da população fraturada, devido a acidentes de trânsito, internada na Santa Casa de São Paulo. Psicologia Hospitalar [ periódico na Internet]. 2004 dez. [acessado 2013 set 5]; 2(2): [cerca de 6p.]. Disponível em: http://pepsic.bvsalud.org/ scielo.php?script $=$ sci_arttext $\&$ pid $=S 1677$. $74092004000200008 \& \operatorname{lng}=$ pt\&nrm=iso.

27. Cordeiro R, Vilela RAG, Medeiros MAT, Gonçalves CGO, Bragantini CA, Varolla AJ. O sistema de vigilância de acidentes do trabalho de Piracicaba, São Paulo, Brasil. Cad Saude Publica 2005; 21(5):15741583.

28. Lipton B, Porter K, Nelson G. Workers compensation temporary total disability indemnity benefit $d u-$ ration. Erlanger: Actuarial \& Economics Division, NCCI; 2010.

29. Laaksonen M, Martikainen P, Rahkonen O, Lahelma E. Explanations for gender differences in sickness absence: evidence from middle-aged municipal employees from Finland. Occup Environ Med 2008; 65(5):325-330. 
30. Souza NSS, Santana VS. Fatores associados à duração dos benefícios por incapacidade: um estudo de coorte. Rev Saude Publica 2012; 46(3):425-434.

31. Santana VS, Araújo-Filho JB, Silva M, Albuquerque-Oliveira PR, Barbosa-Branco A, Nobre LCC. Anos potenciais de vida perdidos e incidência de acidentes de trabalho na Bahia, Brasil. Cad Saude Publica 2007; 23(11):2643-2652.

32. Benavides GB, Benach J, Mira M, Sáez M, Barceló A. Occupational categories and sickness absence certified as attributable to common diseases. Eur J Public Health 2003; 13(1):51-55.

33. Ildefonso SAG, Barbosa-Branco A, AlbuquerqueOliveira PR. Prevalence of temporary social security benefits due to respiratory diseases in Brazil. J Bras Pneumol 2009; 35(1):44-53.

34. Cockell FF, Perticarrari D. Retratos da informalidade: a fragilidade dos sistemas de proteção social em momentos de infortúnio. Cien Saude Colet 2011; 16(3):1709-1718.

Artigo apresentado em 29/11/2012

Aprovado em 20/02/2013

Versão final apresentada em 10/03/2013 